\title{
The Provision of Induction Activities for Imperial College London's International Collaborative PhD Students
}

\author{
Helen Challis, Imperial College London
}

Personally, I think it is always going to be difficult as we only spend short periods of time in both institutions (Imperial College London International Collaborative PhD Student)

\section{Acknowledgement}

This paper originated as a student project from the Post Graduate Certificate in Enhancing the International Student Experience.

\section{Introduction from Dr Dave Burnapp (Course Leader)}

This article addresses issues relating to a relatively new aspect of the internationalisation of higher education. Imperial College London is increasing the number of strategic partnerships it is involved with, and with several of these partners it offers joint and split $\mathrm{PhD}$ opportunities. These students spend parts of their time at each of the partner institutions, so this form of provision raises new issues concerning induction. The findings of this article suggest changes which can be applied more widely.

\section{Abstract}

Imperial College London ${ }^{1}$ currently has five international collaborative $\mathrm{PhD}$ programmes which require students to divide their time between Imperial and a partner institution in Singapore, Malaysia or Hong Kong. International collaborative PhD students frequently arrive for their periods at Imperial at varying times of the year, and are therefore unable to participate in conventional 'Welcome Week' induction activities which occur at the start of the academic session. This article reviews the induction experiences of Imperial's current international collaborative $\mathrm{PhD}$ students, and uses these experiences to reflect upon both the significance of university induction for international students, and, at a more practical level, the form and content of a successful induction process. Using an e-questionnaire, all 30 current international collaborative $\mathrm{PhD}$ students were asked for feedback on their induction experiences, and for suggestions as to how these experiences could have been improved. Findings raised interesting questions as to whether a dual-location programme of study prevents students from feeling a sense of 'belonging' or 'ownership' with regards to their institutions, and whether ongoing orientation can help to ameliorate this. The survey results also indicated that existing induction processes are inadequate, with more assistance being required in the areas of accommodation and immigration support. Drawing on student feedback and the previous work of researchers such as McKinlay et al (1996), the article proposes eight 'action points' for improving international collaborative $\mathrm{PhD}$ students' induction experiences.

Keywords: collaborative $\mathrm{PhD}$; postgraduate induction

${ }^{1}$ Hereafter abbreviated to 'Imperial' 


\section{Research Aims}

This paper focuses on stage 1 of a two-stage project which ultimately aims to develop, implement, and subsequently measure the impact of a successful induction process for students studying on one of Imperial College London's five international collaborative $\mathrm{PhD}$ programmes. The foci at stage 1 was a review of existing students' experiences, their suggestions as to how the induction process could be improved for future students, and the formation of eight recommendations to be considered at stage 2 of the project (not included in this article) when the new induction process is implemented. The review of existing provision took the form of a survey of current students; as Lord and Dawson intended in their research, I hoped to establish 'the nature of the specific enculturation needs' $(2008$, p.2) of these students so that these needs can be met in a more creative and satisfying way. Induction into a university raises interesting questions about institutional 'belonging' and a sense of inclusion. Does this specific group of students perceive the dual-location aspect of their $\mathrm{PhD}$ programme to be a barrier to adapting to life at Imperial, and how could formalised induction - or ongoing orientation - processes help to ameliorate this? Do they have more extensive orientation needs, given that they 'arrive' at Imperial several times over the course of their PhD? Do they still feel as though they are a part of Imperial's community, even when they are based at their home institutions in Southeast Asia? The questionnaire explored these and more practical questions in an attempt to determine the necessary form, content and frequency of induction activities for this unique group of students.

\section{Background to the research}

Imperial is engaged in a continuous process of 'internationalisation' in its many forms. A major strand of its internationalism is the development of strategic partnerships with key overseas institutions. These partnerships have resulted in the creation of split and joint $\mathrm{PhD}$ programmes which are offered in conjunction with overseas partners.

\subsection{Imperial's International Collaborative PhD Programmes}

Imperial currently offers five formal international collaborative (split/joint) $\mathrm{PhD}$ programmes with partners in Southeast Asia. Of these five, two are split programmes, and three are joint PhDs. In this context, the difference between a split and joint $\mathrm{PhD}$ programme is minimal and refers simply to the institution conferring the final degree: for split PhD students, it is solely an Imperial award; for joint PhD students, the award is conferred jointly, by both Imperial and the overseas partner.

The two split programmes are conducted in conjunction with institutions in Malaysia and Singapore. The Malaysia Imperial Doctoral Programme (MIDP) is open to Malaysian nationals only, and is a collaboration with the Malaysian Ministry of Higher Education. Students on this programme split their time between Imperial (for periods of between 3 and 9 months) and one of five partner universities in Malaysia. The A*STAR Imperial Programme (AIP) is a collaboration with A*STAR in Singapore, the leading government agency for scientific research. This programme - for Singaporean nationals only - involves students spending periods of roughly one year in each location. Imperial's three joint $\mathrm{PhDs}$ are very new and, at present, students are only studying on one of the programmes, the joint $\mathrm{PhD}$ with Nanyang Technological University (NTU) in Singapore. The four students currently studying on this programme are of mixed nationality but have all entered the programme via NTU, not Imperial. The other two joint PhDs are with the National University of Singapore (NUS) and the University of Hong Kong (HKU). The number of students studying on Imperial's international collaborative $\mathrm{PhD}$ programmes is listed below: 


\begin{tabular}{|l|c|}
\hline $\begin{array}{c}\text { International Collaborative PhD } \\
\text { Programme }\end{array}$ & $\begin{array}{c}\text { Number of current students } \\
\text { (as at 31 Dec 2010) }\end{array}$ \\
\hline AIP & 14 \\
\hline MIDP & 12 \\
\hline Imperial-NTU Joint PhD & 4 \\
\hline Imperial-NUS Joint PhD & 0 \\
\hline Imperial-HKU Joint PhD & 0 \\
\hline \multicolumn{2}{|c|}{ Number of students studying on Imperial's international collaborative PhD programmes, as at December 2010} \\
\hline
\end{tabular}

All five programmes require students to be physically present at Imperial for part of their $\mathrm{PhD}$, and to be studying at their 'home' institution in Asia for the remainder. In some respects, these students can be considered 'doubly international': not only are they from countries other than the UK - Malaysia/ Singapore/ Hong Kong - but they are also studying on programmes which, due to the dual-location element, could prevent the students from developing a sense of 'belonging' in relation to Imperial. Students frequently arrive at Imperial at various non-conventional times of the year - often having provided little notice of their arrival - and are expected to commence their research immediately. Despite this, they have extensive needs: a formal induction, assistance with accommodation, sometimes language help, and support in joining the student community, both inside and outside the lab.

\subsection{Existing induction support provision}

Imperial has one formal student intake per year, and all undergraduate (UG) and taught postgraduate (PGT) students begin their courses at the start of the academic year in October. An annual international students' 'Welcome Week' takes place at the end of September and includes events such as: a lecture series (on topics including visas, 'London on a Budget', working during studies), scheduled social activities, an Imperial clubs and societies fair, Student Union events, campus tours, and more. The timing of Welcome Week is suited to UG and PGT students, but not PhD students who begin their studies at a different time of the academic year. Given the importance previous research attributes to a successful orientation process, and the status of these students as a group which is potentially 'doubly' international and 'arrives' multiple times during the course of their $\mathrm{PhD}$ programmes, change is required in the form of a newly-developed, specific, tailored induction process for these students.

\subsection{Reason for change}

I have recently taken up the role of manager of Imperial's international collaborative programme portfolio and have decided to review induction processes. This activity has been informed by a March 2010 report by Universities UK which highlighted the importance of not homogenising international students and of providing 'customised services appropriate to different student groups' (Archer et al., 2010, p.13). The current lack of sufficient induction support mechanisms for Imperial's international collaborative $\mathrm{PhD}$ students has been formally noted by senior management in the programmes' academic board meetings, and a need has arisen for split/joint PhD students to be granted the same level of service as other Imperial students. Aside from the obvious benefit to the students of feeling adequately inducted and included in the Imperial community, a lack of sufficient orientation provision could carry severe reputational risks for Imperial if unhappy students report back to their home institutions and international peers. The number of students studying on the international split $\mathrm{PhD}$ programmes has been relatively small to date, at around 40 students over a 5-year period. However, with the recent launch of the three joint $\mathrm{PhD}$ programmes, this number is set to significantly increase. The increase in Imperial's international 
collaborative $\mathrm{PhD}$ provision, coupled with my new role, means I am in a position to establish the specific induction needs of this unique and specialised group of students.

\section{Literature Review}

Whilst, broadly speaking, international students' opinions about induction processes have been widely documented, there has been little written about the experience of split/joint $\mathrm{PhD}$ students, most likely because these students' situations are relatively uncommon.

A clear message from relevant literature is that adequate induction is a necessity (Green $e t$ al., 2008; Archer et al., 2010). Universities UK suggests it 'can make a world of difference in how the initial experience [of the university] is perceived' (Archer et al., 2010, p.23). Lord and Dawson expand this to suggest the existence of a link between successful orientation and retention of students (2008, p.3), and though this claim is not substantiated with data, it is also highlighted by UKCISA as a possible welcome consequence of induction (2008, p.12). In addition to improving student wellbeing and retention, satisfied students could function as university ambassadors upon return to their home countries. Biggs and Tang suggest universities have become increasingly 'corporatized and competitive for markets' (2007, p.2). If this is the case - and it seems likely, given the $£ 2.9$ billion cut to universities' funding announced in October 2010 - the 'customer's' initial experience becomes key: a positive experience could result in a (free) ambassador for the institution; a bad experience could mean the opposite. This is a situation recognised by the International Student Barometer (ISB) which includes the provision of orientation activities in its benchmarking. The importance of keeping students 'on side' should not be underestimated; as Mazzarol and Soutar identify, 'word-of-mouth referral is one of the most powerful forms of promotion that international education institutions can use' $(2002, \mathrm{p} .85)$. This extends beyond students, to also encompass the views of parents, friends, previous teachers etc. This is particularly pertinent to this project, given the newness of the programmes under review and the lack of staff dedicated to the programmes' development. Accordingly, at this stage, Imperial is largely reliant on student word-of-mouth as a method of recruiting new students.

The proliferation of induction/acculturation 'guides' - for both students and for institutions suggests that a successful induction is recognised by universities as productive. An example is the 'Prepare for Success' website, an online toolkit of interactive learning to help students prepare for arrival in the UK. A look at the website's user statistics reveals that Hong Kong, Malaysia and Singapore all feature in the top 20 (Prepare for Success, 2010) in terms of countries from which the website receives most 'hits', a statistic which highlights students' desire and demand for pre-arrival support. There are similar manuals for universities that provide advice about formulating a successful induction programme, an example of which is UKCISA's comprehensive Planning and Running Orientation Programmes for International Students (Green et al., 2008). This guide covers every element of orientation programmes, including: planning, delivery, resources, and ongoing orientation. These codes of practice will be considered later in this article and will inform stage 2 of this project.

Another key point arising from existing literature is the importance of avoiding homogenisation by providing specific, considered services for different student groups. Clearly, there is no one model for successful orientation. Some researchers, such as McKinlay et al, found that pre-sessional induction actually increased the prevalence of homesickness and culture shock among students, attributing this to orientation 'creat[ing] a false sense of security' (1996, p.388). Despite this, they offer a list of recommendations for relevant university staff. I repeat these recommendations in full here: 
- A more sophisticated analysis of the problems and needs of individual international students, relevant to the local higher education environment.

- The development of coherent management strategies to support international students. Support is often provided by several agencies within institutions in a relatively uncoordinated and unstructured way. Communication between these agencies, and between them and students, tends to be poor.

- A support system that would address student needs all year round.

- A support system that reaches those who do not participate in initial orientation programmes. The assumption tends to be made that students not participating in initial programmes do not feel they need support. However, many students are not able to participate in such programmes for reasons outside their control.

- The development of good documentation of the support system, so that international students know where to go for help and advice, and the provision of accurate information about the host environment.

- Less emphasis on the integration of international students, who will remain in a host country for a relatively short time, and an encouragement to maintain links with home, and form links with co-nationals in the host country.

My initial work on this project, in the form of an assessment of financial and staff resourcing, has shown me that McKinlay et al's suggestions would - if appropriate - be achievable in the context of Imperial's international collaborative $\mathrm{PhD}$ programmes. Their comprehensive recommendations as listed above will therefore be used as a framework with which to consider the findings from this article's survey.

\section{Research Methodology}

An e-questionnaire containing a mixture of closed and open questions was emailed to all 30 current split/joint PhD students. The questionnaire was prefaced by a brief explanation as to the purpose of the questions, and a reassurance of anonymity. At the time of research, many students were overseas; accordingly, I selected email as the most satisfactory mode of communication. I could contact all students, regardless of location and irrespective of time zones, and email allows respondents time to consider their answers. Green et al. highlight the email nous of students, suggesting: 'internet access can be the most important thing to new students' (2008, p.66). Similarly, current Imperial students are familiar with email as a principal mode of communication.

Questions 1-3 were closed questions with which the intention was to establish certain key facts such as when the student started their PhD. This was relevant as it would reveal whether students started their programme during or outside Imperial's annual Welcome Week in September/October.

The subsequent research questions were focused around four key themes: pre-arrival support, post-arrival support, social life, and a sense of institutional 'belonging'. The questions were as follows:

4. Before starting the programme, did the student receive information about the facilities/services available to postgraduate students at Imperial?

5. Where did this information come from? (International Office, Registry, Department)

6. Of the items listed in question (4), how helpful did the student find this information?

7. With the benefit of hindsight, how could this pre-arrival information be improved? 
8. When the student came to London for their first London-based period of study, did the student receive any welcome/orientation? This may have been organised by the student's department, or the International Office.

9. Does the student consider themselves a regular user of Imperial facilities such as: gym, cafes/restaurants, Student Union, clubs and societies?

10. How did the student hear about these facilities?

11. Has the student made friends at Imperial with students who are not studying on a split/joint $\mathrm{PhD}$ programme?

12. The student is required to split their time between two institutions in different countries. To what extent does the student think it is possible for students studying on split/joint $\mathrm{PhD}$ programmes to feel a part of the community of both institutions?

13. If the student was asked by a stranger which university they attended, what would their answer be?

Answers to questions 4-8 will be used to inform the development of a new induction process at stage 2 of this project. Questions 9-11 were intended to reveal the extent to which split/joint PhD students become a part of the 'community' of Imperial by enquiring about usage of the university's social facilities - cafes, clubs, gym etc - and whether they have friends studying on full-time, Imperial-based PhDs. Clearly, a sense of membership of an institution's community is likely to transcend simple facts such as the number of societies a student is a member of. For this reason, I included questions 12 and 13 which I hoped would reveal these students' thoughts about the possibility of studying in multiple institutions whilst still feeling a sense of belonging and 'ownership' towards both.

I am focusing on student opinion for the obvious reason that it is they whom these processes directly affect, but also because my reading has shown me that exceptional international student voices are sometimes omitted in favour of a singular, apparently universal, 'international student voice'. In her book Internationalisation and the Student Voice, Elspeth Jones outlines the importance of attempting to capture a breadth of student voices, and not simply focusing on conventional groupings and dichotomies such as postgraduate/ undergraduate, home/ international $(2010$, p.xv). This piece of research attempted to tackle this convention by surveying a unique group of students who have not yet been 'heard'. Jones argues that this is a necessary process in order to 'produce the graduate for the twentyfirst century' (2010, p.xv). These students are studying acutely international programmes and are, in a sense, pioneers. It seems important, therefore, that their voices are captured now, at this formative stage.

One hindrance of which I am aware is the potential reluctance of students to be forthcoming with their truthful opinions. As Chan articulates, some (South)East Asian students particularly those from China - 'prefer not to express their true opinions so as not to embarrass or offend others' (Chan, 1999, p.299). However, given the circumstances, I am still of the view that email was the most appropriate mode of communication. Face-to-face questioning seems somehow more forceful, and could have increased the likelihood of students being reticent due to fear of causing offence.

\section{Findings}

There was a $47 \%$ response rate with 14 of the 30 current students responding.

Respondents included: 4 from the MIDP split $\mathrm{PhD}$ (27\% response rate), 7 from the AIP split $\mathrm{PhD}(58 \%)$, and 3 from the NTU joint PhD programme (75\%). PhD start dates ranged from December 2006 to December 2010, meaning the full cross-section of current PhD students - 
both early and late-stage - are represented by the responses. Eight of the respondents started their $\mathrm{PhD}$ programmes overseas at Imperial's international partners, and 6 began their programmes based in London. The survey's findings are presented here under the four headings of pre-arrival induction support, post-arrival induction support, social life, and institutional 'belonging' or 'ownership'.

\subsection{Pre-arrival support}

Nine of the 14 respondents received either none or very little information about nonacademic postgraduate facilities before their arrival at Imperial. These students tended to ask their friends for advice, or refer to Imperial's website. There was consistency in the materials provided to the 5 students who did receive pre-arrival induction information. This tended to consist of a London tube map and guide, transfer exam criteria, International Office staff contact details, and information about clubs and societies. These students received this information from the International Office, a discovery which highlights the importance of my office's role in preparing students for their arrival in London. Only 3 students received any pre-arrival information from the Registry, and 2 received information from their Imperial department. The centrality of the International Office's role in providing this pre-arrival support is confirmed by the comment of one MIDP student who stated: 'No, I did not receive much information. The previous guy in charge from the International Office was about to leave Imperial'.

Although only 6 students claimed to have received any pre-arrival information, 8 students stated they were satisfied with the pre-arrival information received. This suggests that prearrival support is less important to some students than others. Two students were satisfied with some of the information they received but felt it was too basic. The remaining 6 students provided positive feedback, with one AIP student stating:

I also got a package introducing various personnel (academically and life-related), who will be of great help when I encounter problems in the future. I feel very assured and confident to know these people. In addition, I received information about Imperial College club fair [...] which help me to mingle with different people and appreciate a different culture.

(AIP student, 2010)

When asked how pre-arrival information could be improved, 10 of 14 respondents referred to a need for increased support with finding short-term accommodation. This was not surprising; given students' frequent arrivals at unconventional times of the academic year, university halls of residence are not usually available for their use and they are required to find privately-let accommodation. A need for better pre-arrival immigration support was also highlighted by 8 of 14 students. Pre-arrival accommodation and visa advice were two areas which were almost unanimously highlighted as needing attention.

Respondents were forthcoming in offering suggestions as to how the pre-arrival service could be improved. Ideas included: the provision of a list of available accommodation; a checklist regarding the matriculation process; advice about applying for an Oyster card [for travel on London transport]; having regular socials with other scholars on split/joint $\mathrm{PhD}$ programmes; emailing maps in advance; providing information earlier; and a fully comprehensive guide to being a student in London. An interesting suggestion was made by an AIP student who hinted at the potential loneliness faced by international split/joint PhD students: 
Getting all scholars connected and give a regular update on which scholars are currently in London and Singapore would give the scholars coming to London assurance that they are not alone.

(AIP student, 2010)

\subsection{Post-arrival support}

One respondent omitted to answer the question enquiring whether or not they received any welcome/orientation upon arriving in London. The remaining 13 respondents all received some form - however variable - of post-arrival induction. Seven students received a welcome from International Office staff, and 7 students were also formally inducted by their Imperial department. Some students received only the minimum, practical, laboratory safety course, but others participated in a comprehensive, considered induction process:

Yes, at the college, my supervisor assigned a student to orientate me to the facilities in my building and laboratory and whom I should look for if I needed various things, such as ordering chemicals. My supervisor also brought me to speak to the post graduate administrator of my department (Bioengineering), who gave me a welcome package with the campus map, information on activities which are taking place during the welcome week, such as the Fresher's Fair. This was informative and I felt welcomed. We also had a Welcome Drinks session where I got to meet the other new students and people in the department. I feel that by providing students with information and organizing a series of activities for students to participate in, they can feel more at ease in a new environment and make more friends.

(NTU student, 2010)

The feedback suggests that those students who arrived close to Welcome Week at the beginning of the academic year were more satisfied with the induction they received as the university as a whole was prepared for students' arrival and the students could effectively 'piggyback' onto university-wide events.

\subsection{Social life}

Six of 14 respondents do not consider themselves regular users of Imperial facilities such as the gym, cafes, the Student Union, and clubs and societies. One reason offered for this was the students' location at one of Imperial's hospital campuses as opposed to the main campus at South Kensington where many of the facilities are located. The other 8 students regularly eat in Imperial cafes and use the gym. Four students are members of Student Union clubs, often choosing those relevant to their nationality. Examples included the Singapore society and the Wing Chun (Chinese martial arts) club. Respondents highlighted the importance of their peers in finding out about these facilities and social opportunities; of 14 respondents, 8 named friends as the source of information. Other significant channels of communication included supervisors and the International Office.

\subsection{Institutional 'belonging'}

When asked whether they had made friends with students studying on non-split/joint $\mathrm{PhD}$ programmes, only 1 of 14 students answered 'no'. This could be explained by the student's recent - December 2010 - start date. The other 13 respondents all answered positively, listing clubs, societies, and lab peers as good sources of friends.

Question 12, asking whether students felt it was possible to feel part of the community of multiple institutions, prompted some interesting responses. Some respondents interpreted the question as referring to an academic community, and some read it as a reference to social 
lives. Five students highlighted the significance of regular communication between the Imperial and overseas supervisor as being key to a student's sense of wellbeing. Four students felt that responsibility for feeling included in an institution's community lay with the student: 'It takes time. It's entirely up to the student to interact with the lab people' (AIP student, 2010). Six students highlighted the length of each period of time spent at the different institutions as being relevant, suggesting that if a student splits their time equally between their two universities, they are more likely to feel a part of both communities. If a student spends short periods of time at Imperial (as is the case for many MIDP students), they are less likely to feel included in Imperial's community and to view the university as 'their' institution. There were variations in the importance attributed to a sense of inclusion in Imperial's community, with one MIDP student stating:

Being a PhD student, there are less social lives involved, life is more focused on the research and most of time is spent in the lab.

(MIDP student, 2010)

Answers to the questionnaire's final question, in which students were asked how they would respond if a stranger asked them which university they attended, were not entirely unexpected, with split PhD students - 9-giving Imperial as their answer. This could be explained by the fact that split $\mathrm{PhD}$ students graduate with an Imperial-only $\mathrm{PhD}$. Joint $\mathrm{PhD}$ students stated that they would name both institutions in their answer, given that they graduate with a joint $\mathrm{PhD}$ award conferred by two universities. Some respondents were clearly pleased to be an Imperial student: 'Imperial College London, that's for sure. I am proud to be part of Imperial' (AIP student, 2010). One MIDP student gave a particularly detailed response:

A stranger in London: Imperial College. A stranger in Malaysia: My Malaysian host university. It's weird to provide a long answer [...] However, I'm definitely happy to give the long answer to my friends or academicians, every time they asked. Not everyone is eligible to answer like that, right? So I'm lucky!

(MIDP student, 2010)

Being physically present in London was revealed as being crucial to students feeling as if they 'belonged' to Imperial's community. Several respondents echoed the MIDP student above in stating that they would name the university located in whichever country they were in when asked the question. This suggests that more support could be provided in the form of ongoing orientation activities which could help to foster a sense of inclusion, even whilst physically absent. Split and joint PhD students are entitled to full access to all Imperial facilities for the duration of their PhDs, regardless of their location. It is important, for reasons of equality and fairness, that international collaborative $\mathrm{PhD}$ students feel as able to maximise their time as Imperial students as those studying on conventional $\mathrm{PhD}$ programmes. It would be unfortunate for these students to forego potentially significant academic and social opportunities simply due to a feeling that they do not 'belong' to Imperial during their overseas periods.

\section{Discussion}

The intention with the student survey was to gather information which would inform the development of a new induction process for split/joint $\mathrm{PhD}$ students. I also wanted to explore whether the dual-location aspect of these students' PhD programmes prevented them from feeling 'at home' during their time at Imperial. Both of these issues will now be considered in turn, before compiling a list of 'action points' which will inform stage 2 of the project. 


\subsection{Development of new induction process}

Starting by positing the question 'does induction matter?', the answer from the students is a resounding 'yes', with every respondent suggesting ways pre-arrival information could be improved. In terms of the provision of pre-arrival information, use of email and the web seems an acceptable method of delivery, with 8 students mentioning that they had, unprompted, searched the Imperial website for relevant information.

In terms of the content of pre-arrival information, two dominant themes were visa advice, and accommodation support. Accommodation is an area which has been highlighted in senior board meetings as needing attention, so the survey's findings reinforced this message. UKCISA's Managing Accommodation for International Students: a Handbook for Practitioners could be useful here. Imperial's accommodation system is ill-equipped to accommodate students researching at the university for periods of less than a semester, something beyond the control of the International Office. This is a prime example of the issue highlighted by McKinlay et al, of fragmented and uncoordinated support agencies (1996, p.392). To ameliorate this, the International Office needs to provide more support in the form of lists of reputable letting agents and private sector halls of residence, so that students are able to secure accommodation pre-arrival. Visa advice is also managed by my office, the International Office, and I think it would be useful for me to refresh my immigration training so that I am in a position to provide quick advice to students. The importance of students registering with the police upon arrival in the UK also needs to be emphasised:

I think it will be great if the information also include a reminder for police registration [...] I almost missed it when I came to London

(AIP student, 2010)

Student responses highlighted the lack of consistency in terms of the office administering pre-arrival information. Some responsibility must lie with academic departments, but the International Office could provide a welcome information pack earlier on. Situations such as 'information was given quite late and thus procedure had to be rushed' (NTU student, 2010) should be easily avoided by better monitoring of arrival and admissions. This can be achieved by liaising more closely and consistently with the Registry department, perhaps via a monthly summary email. Students who had the opportunity to meet with an International Office representative upon arriving in the UK for their first research period seemed more satisfied with their induction:

\section{I had a welcome chat by Joe Bloggs from the International Office, who gave me a campus} tour and clarified some of the queries I had

(AIP student, 2010)

Given the small number of students on these programmes to date, a personal meeting with all new arrivals in London should be made a requisite. Similarly, it should be made clear at this meeting that International Office staff are available to assist post-arrival, too.

McKinlay et al emphasise the importance of:

The development of good documentation of the support system, so that international students know where to go for help and advice, and the provision of accurate information about the host environment.

(1996, p.392)

Students were forthcoming in making suggestions about what this 'good documentation' might include. Most are listed above, but generally included: visa and accommodation 
support; London travel information; directions of how to reach accommodation from the airport; budgeting estimates; exact location to obtain matriculation card; details about the private accommodation market including how to pay a deposit; how to set up a bank account; and a 'one-stop-shop' booklet in which this information is available. Using the information provided, stage 2 of this project will include the development of an e-handbook ('good documentation of the support system') which will be created and updated by the International Office. This will capture information and links to relevant websites of concern to split/joint $\mathrm{PhD}$ students. It will also include a clear list of contacts which should function as a signpost revealing who to contact in a variety of situations.

\subsection{Institutional 'belonging'}

Respondents generally felt it was possible to feel a sense of inclusion at two institutions, but that this required work on the part of co-supervisors, students, and peers. Half of respondents mentioned schemes which would be considered part of post-arrival support, or ongoing orientation. McKinlay et al (1996) recommend the implementation of a support system that addresses student needs all year round (p.392). This can help to counteract or even prevent students' late-onset periods of insecurity. Respondents provided suggestions, including: a buddy scheme to assist with non-academic support; more scheduled socials for split/joint $\mathrm{PhD}$ students to attend; and a regular update email to all collaborative $\mathrm{PhD}$ students about the location of scholars, whether at Imperial or overseas. The latter two suggestions are easy to achieve, and there is a budget for increased social activities. A buddy scheme would require greater resourcing and consideration, but could be a possibility.

It is important that any ongoing orientation activities are accessible to all students, regardless of which campus they are based at ('No, I have not gotten a chance to use facilities as I am currently based in Hammersmith' (AIP student, 2010)). It should also be emphasised at the initial meeting that orientation will be ongoing and doesn't 'stop' after two weeks. Given that these students undergo the process of 'arrival' multiple times during the course of their $\mathrm{PhD}$ programmes, ongoing orientation plays a significant role in aiding a sense of institution ownership: 'If the environment makes them feel welcomed and not treat them as a mere visitor, they will feel part of the community' (NTU student, 2010). Students seem to be traversing the barrier of being the 'other', or visitor, with success: thirteen of fourteen students feel they have made friends with students studying on other $\mathrm{PhD}$ programmes. One of the methods by which students have engaged with Imperial's community is by joining student societies: 'I would like to involve in some extra-curricular activities outside the scientific lab, which help me to mingle with different people and appreciate a different culture' (AIP student, 2010). Some of these societies indicate students are socialising with people of the same nationality (Singaporean Society etc), but are also meeting students from other countries: 'It is important to not always socialise with only people of your own nationality while in London, because that defeats the purpose' (AIP student, 2010). Perhaps, therefore, the International Office could think of strategies by which to involve students of other nationalities in the collaborative students' inductions. The UKCISA guide suggests students' sense of community and belonging can be helped if they can meet others from: their own country, their course, those they will be living near, and those that they might not talk to by chance (p.40). Again, the idea of a buddy scheme, whereby international collaborative students are paired with non-collaborative $\mathrm{PhD}$ students, could be relevant here.

An eagerness to engage with UK culture is not unanimous; for the MIDP students in particular, there seemed to be a limited interest in socialising. This resonates with McKinlay 
et al's recommendation that students who are in the host country for a short time (as is the case for MIDP students) should be actively encouraged by institutions to 'form links with conationals in the host country' (1996, p.392). Some students view the purpose of their time at Imperial as being to simply study: 'life is more focused on the research' (MIDP). McKinlay et al reiterate this scenario, suggesting: 'the student [...] is a sojourner and will stay only temporarily in the host culture [...] therefore preoccupied with the academic task in hand' (1996, p.391). Rather than trying to change this by creating compulsory ongoing orientation events, it seems more logical to be accepting. As the article explains, students in the UK for short periods of time are wise to maintain a degree of distance from the host culture as they are 'therefore more able to assimilate back into their own culture with as few problems as possible' (1996, p.380). This suggests that an ongoing orientation programme would be of more interest to AIP and joint $\mathrm{PhD}$ students who spend periods of at least one year's duration at each institution.

\section{Action Points: eight recommendations to be considered at Stage 2}

- International Office to develop the collaborative programmes section of the Imperial website. Students expect and want to find information online, in a one-stop-shop format. Information provided should include: accommodation, immigration, life in London, $\mathrm{PhD}$ milestones, budgets, and key contacts. The International Office should be emphasised as principal contact for any non-academic issue.

- Same information to be compiled in a non-generic e-handbook to be emailed to students within two weeks of their acceptance onto the $\mathrm{PhD}$ programme, regardless of their start location.

- Where possible, students should be encouraged to time their first arrival at Imperial with Welcome Week.

- All international collaborative PhD students arriving at Imperial should meet with an International Office staff member within their first week. Taking into account that students are unlikely to read or absorb all information provided in advance, new students should be provided with a hard copy of the e-handbook. Meeting should take the form of an informal chat over coffee, followed by a campus tour.

- Regular and varied programme of social events for international collaborative $\mathrm{PhD}$ students, organised by the International Office. There is a budget for an event every three months: format should be decided by students, but could take the form of a meal, bowling, a small trip etc.

- More frequent, informal meet ups in addition to the quarterly events. These should be lower cost and could take the form of: a picnic at lunchtime, or attending an Imperial concert together. The location of these should be varied so that students based at hospital campuses do not always have to travel to South Kensington. Can be dictated by demand but one event per month seems possible, given students' willingness in feedback.

- Participation in ongoing orientation events should be made more attractive by the provision of refreshments. This strategy brought success in December 2010 when the International Office organised a well-attended Christmas dinner for London-based students.

- A monthly update email to be sent to all collaborative $\mathrm{PhD}$ students with details as to who is in which country. This should facilitate greater, cross-programme communication. 


\section{Research Reflections}

This study was investigative, seeking to gather information which could inform a later stage of the same project. Its success lies in capturing current students' voices and thus making them the architects of future students' orientation experiences. Responses to the survey reveal a suggested framework which resonates with that proposed by McKinlay et al. Certainly, as was the intention of the survey, progress has been made in determining the specific enculturation needs of this unique group of students, but there is scope to 'drilldown' and follow-up with some interviews, perhaps focused particularly on the issue of 'owning' an institution. How can a 'dual' student feel both 'here' and 'there'? What can we - support staff - do to help enable students have this sense, both emotionally and practically? Should time permit, further investigation into both academic and administrative staff's view of provision for collaborative students could prove insightful. However, the results of this stage 1 of this project - have provided a solid basis upon which to build an enhanced induction and orientation process for a unique but needful group of students.

\section{References}

Archer, W., Jones, E. and Davison, J. (2010) A UK Guide to Enhancing the International Student Experience. London: International Unit, Universities UK

Biggs, J. and Tang, C. (2007) Teaching for Quality Learning at University. Maidenhead: Open University Press

Chan, S. (1999) The Chinese Learner - A Question of Style. Education and Training. 41, (7), 294-304.

Green, D. and Healy, L. (2008) Planning and Running Orientation Events for International Students. London: UKCISA

Jones, E. (2010) Preface. In: Jones, E. (ed.) Internationalisation and the Student Voice. New York: Routledge. pp.xv-xxv.

Lord, P. and Dawson, C. (2008) The Induction Needs of International Students at Postgraduate Level. Higher Education Academy [online] Available from: http://www.1las.ac.uk/materialsbank/mb080/LO_3/lord_business_sc.pdf [Accessed 20 November 2010]

Mazzarol, T. and Soutar, G. (2002) "Push-pull" factors influencing international student destination choice. The International Journal of Educational Management. 16, (2), 82-90.

McKinlay, N., Pattison, H. and Gross, H. (1996) An Exploratory Investigation of the Effects of a Cultural Orientation Programme on the Psychological Well-Being of International University Students. Higher Education. 31, (3), 379-395.

Prepare for Success (2010) Visitors to $\underline{w w w . p r e p a r e f o r s u c c e s s . o r g . u k}$ [online] Available from:

http://www3.clustrmaps.com/counter/maps.php?url=http://www.prepareforsuccess.org.uk [Accessed 18 November 2010] 


\section{About the author}

Helen Challis is International Officer (Strategy and Partnerships) at Imperial College London.

Email: h.challis@imperial.ac.uk 